\title{
Real gains from flow-based methods for allocating power transmission capacity in Europe
}

\author{
Vincent Rious, Philippe Dessante
}

\begin{abstract}
This paper aims at understanding and assessing the main methods proposed by ETSO and EuroPEX [1] to define and allocate interconnection capacities in Europe. We model these methods and evaluate their technical and economic efficiency on a 7-node network. We find first, that, unsurprisingly, the allocation methods are all the more efficient as the Kirchhoff laws are integrated more precisely in the market clearing. Second, and more surprisingly, we find that the zonal vision of the grid considered by all these methods has important consequences on the technical and economic efficiency as it may cause the capacity limits of powerlines to be exceeded.
\end{abstract}

Index Terms - Electricity, Transmission Capacity Allocation, Market Coupling, ATC-based, flow-based.

\section{INTRODUCTION}

$\mathrm{T}$ HE interconnections are fundamental for the European power market as they are the easiest way to stimulate competition between incumbents [2]. That is why the TSOs, following the intention of the European Commission [3], have proposed the concept of flow-based allocation method that should allow the market participants to use transmission capacities closer to their physical limits. The market coupling in Europe currently relies on the concept of Available Transfer Capacity (ATC) where the physical Kirchhoff laws are partially ignored. This assumption generally prevents from using all physical interconnection capacity and so from benefiting from more competition and efficiency in the power market. Some TSOs in the Central Western Europe plan to implement in some years the flow-based allocation method. This method should overcome the inefficiencies resulting from the concept of ATC as the market for power with the flowbased allocation method is cleared taking into account explicitly the Kirchhoff laws.

The introduction of this new flow-based concept to allocate transmission capacity in Europe nevertheless raises two main questions. First what is precisely the flow-based allocation method? Indeed, currently, no model of either ATC- or flow-

Manuscript received March 8, 2009. This work was supported by the French Regulatory Commission (CRE) under the €nergy Project II.

Vincent Rious and Philippe Dessante are with Supelec, Gif-sur-Yvette, 91190, France (phone: 003316985 1531, fax: $0033 \quad 169851539$, vincent.rious@supelec.fr). based allocation method is publicly available, except their literary descriptions by ETSO and EuroPEX [1]. Second what are the real gains of the flow-based allocation method compared to the current ATC-based method? The Central Western European TSOs and PXs have already compared the ATC- and flow-based allocation methods [4]. This preliminary evaluation results in a quite paradoxical conclusion: the flowbased method might be less efficient than the current ATCbased method.

To answer these two questions, we propose to model, evaluate and compare the ATC- and flow-based methods described by ETSO and EuroPEX [1]. Modelling these methods prompts us to enter in the intimacy of their design. As a consequence, we can better know the steps needed in the different methods to define the interconnection capacity. Moreover, these models allow us to compare the ATC- and flow-based allocation methods, not only measuring social welfare but also checking that these allocation methods respect the physical limits of the power grid.

The aim of this paper is twofold. Firstly this paper proposes models for the ETSO-EuroPEX methods for defining and allocating interconnection capacities in Europe. Secondly this paper assesses the differences of these methods $1^{\circ}$ from a conceptual point of view, from the point of view of its $2^{\circ}$ technical and $3^{\circ}$ economic efficiency.

This paper is organized as follow. In section II we present the assumptions and data we will use to evaluate the ETSOEuroPEX methods. In section III, we present and analyse the similarities and differences of these methods. In section IV, we model these methods and evaluate their technical and economic efficiency. Section V concludes.

\section{ASSUMPTIONS AND DATA}

\section{A. Assumptions}

In this paper, we use the five following technical assumptions. $1^{\circ}$ The DC approximation is used to calculate the power flows. $2^{\circ} \mathrm{We}$ don't consider any N-1 or N-k criteria. $3^{\circ} \mathrm{We}$ focus only on day-ahead exchanges for calculating cross-border capacities. $4^{\circ} \mathrm{We}$ assume the TSOs are perfectly coordinated mimicking a unique TSO for the operation of the whole interconnected network. Therefore we test only the efficiency of each congestion management scheme. $5^{\circ} \mathrm{We}$ 
work with a perfect knowledge of the available line capacities and of the generation and load schedule established before day-ahead. We make also the two following economic assumptions. $1^{\circ}$ The transmission capacities are allocated jointly with the market clearing through an implicit mechanism. $2^{\circ}$ Neither TSO nor generator have market power.

\section{B. Network for simulations}

To evaluate the ETSO-EuroPEX congestion management schemes, we use the network already described in [5] because the authors used this network too in order to test the efficiency of different congestion management schemes. This network with 7 nodes and 10 lines is cut out in 3 zones as illustrated in Fig. 1. The capacity of each powerline is $100 \mathrm{MW}$.

The generators are connected only to nodes $1,3,4,5$ and 7 Their cost functions are the same ones as those used in [5]. A load is connected to each node. We assume that the consumers are not elastic to price. Table I describes their consumption. Total demand in zone 1 (respectively 2 and 3 ) is then $330 \mathrm{MW}$ (respectively $170 \mathrm{MW}$ and $530 \mathrm{MW}$ ).

TABLE I NODAL LOAD

\begin{tabular}{|l|c|c|c|c|c|c|c|}
\hline Node & 1 & 2 & 3 & 4 & 5 & 6 & 7 \\
\hline Load (MW) & 125 & 115 & 170 & 90 & 165 & 190 & 255 \\
\hline
\end{tabular}

\section{Base case}

Generation and load scheduled for every node before dayahead and the resulting line flows describe the base case. A physical margin can then be calculated for each line as the difference between the line capacity and the base case flow. We decide here that the base case corresponds to the situation where the market equilibrium is obtained considering each zone as isolated, that is to say that there is no commercial cross-border exchange (see Fig. 1). Then the market prices are respectively in zone 1, 2 and 3, 31.5€/MWh, $39.8 € / \mathrm{MWh}$, and $63.5 € / \mathrm{MWh}$.

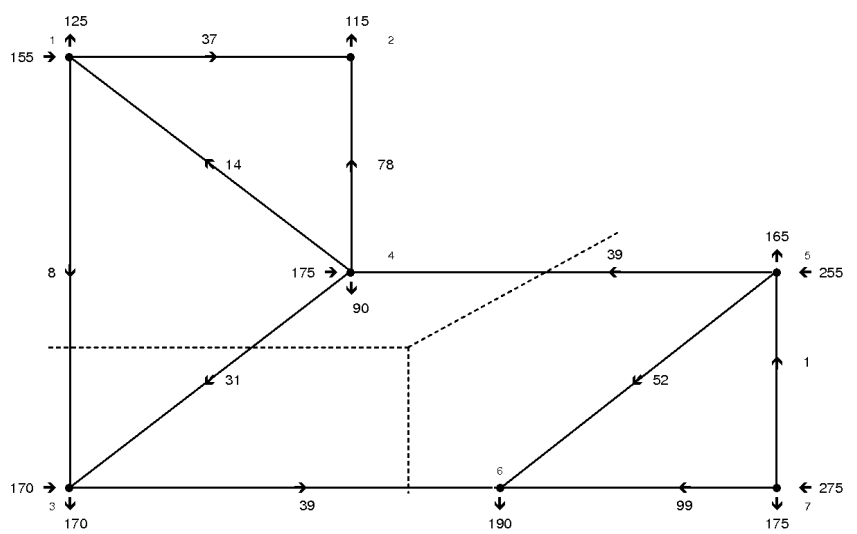

Fig. 1. Base case to test the ETSO-EuroPEX methods

\section{Benchmark: nodal pricing and optimal network use}

To know the optimal use of the network infrastructures, we consider the equilibrium of a nodal market (see Fig. 2).

Line 3-6 is congested. Zone 1 is then exporting $191 \mathrm{MW}$, zone 3 importing $186 \mathrm{MW}$ and zone 2 is slightly importing 5 MW. The social cost is then $30403 €$.

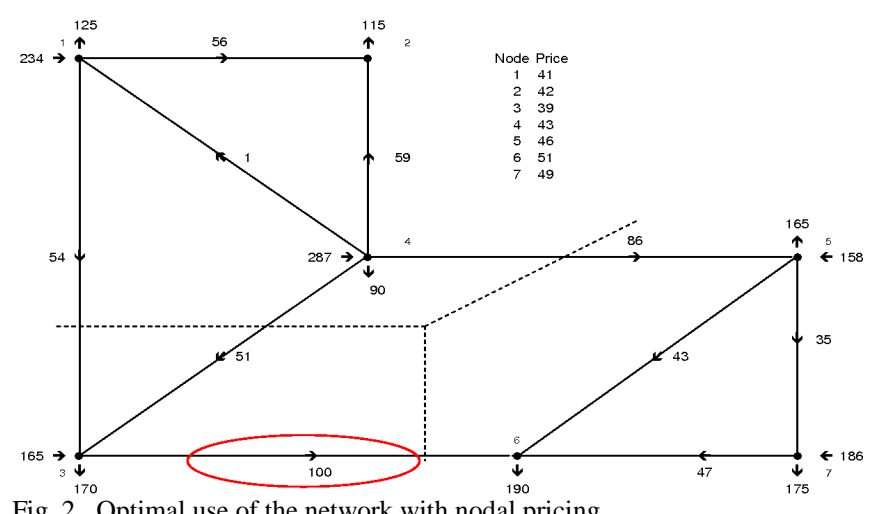

Fig. 2. Optimal use of the network with nodal pricing

\section{Methods TO CALCULATE EXCHANGE CAPACITIES}

In this section, we present the three main ETSO-EuroPEX congestion management schemes (over the five ones proposed). We identify their similarities and their differences.

\section{A. Presentation of the ETSO-EuroPEX methods}

The first main method presented by ETSO and EuroPEX is Available Transfer Capacity (ATC). To ensure security, this method applied on a meshed network takes into account the possible impact of unidentified powerflows resulting by the Kirchhoff laws from transactions between other market zones. It forces the TSOs to display small cross-border capacities compared to their optimal values considering the most constraining situation. This method is widely used for instance on the French borders with Belgium, and Germany [8].

The second main method exposed by ETSO and EuroPEX is "Combined ATC". This method consists in determining some ATC for exchanges related to several borders. This definition allows to offer more important cross-border capacities to the market than with the previous method. The Combined ATC method is used for instance in Germany where there is a joint limit on the exchanges with France, Switzerland, and the Netherlands.

The last main method exposed by ETSO and EuroPEX is exclusively flow-based. The Kirchhoff laws are explicitly considered in the market clearing. It is the Critical Branch flow-based method. In this method, the constraints on the network are not aggregated on the border. Some real powerlines and their physical limits are included in the market clearing because they are critical branches for cross-border exchanges. This method for defining the exchange capacities is currently under study in several regions in Europe because it could offer a maximum of cross-border capacities while ensuring a high level of security for the power system.

\section{B. Similarities and differences in the methods}

The methods exposed by ETSO and EuroPEX to define cross-border capacity have differences and similarities. Identifying them is needed to design an analysis framework for evaluating these methods in similar conditions while respecting their particularities. 
1) A common point for all the methods from ETSO-EuroPEX, the zonal approach

All the ETSO-EuroPEX methods are zonal congestion management schemes. However, it is the nodal day-ahead modification of the generation pattern that creates, through the Kirchoff laws, additional power flows to the base case ones. To calculate the cross-border capacities in the ETSOEuroPEX methods, it is necessary to set an assumption on how the day-ahead increase (respectively decrease) of the zonal net exports are distributed and created by the individual increase (resp. decrease) of generators in each zone.

The nodal sharing of the modification of net export of each zone requires to define a matrix called Generation Shift Keys (GSK). This GSK matrix has $n$ raws and $z$ columns, where $n$ is the number of nodes on the network and $z$ the number of market zones cutting out the network. The transposed matrix in Equation 1 below gives approximated GSK values from the base case to the optimum both presented in II. D. We will use the values in the rest of this paper.

The meaning of the GSK matrix is the following one. Let consider the first raw of this transposed matrix. When there is a change in the net export of zone 1 (for instance an increase by $100 \mathrm{MW}), 45 \%$ of this zonal change comes from generator at node 1 and $55 \%$ of the zonal change comes from generator located at node 4 . This rationale extends to the other zones.

$$
G S K^{\prime}=\left[\begin{array}{ccccccc}
45 \% & 0 & 0 & 55 \% & 0 & 0 & 0 \\
0 & 0 & 100 \% & 0 & 0 & 0 & 0 \\
0 & 0 & 0 & 0 & 50 \% & 0 & 50 \%
\end{array}\right]
$$

ETSO and EuroPEX formalise the concept of a GSK matrix for the Critical Branch method only. At the same time, the GSK matrix generalises two approaches already exposed by ETSO in [6] to calculate ATC. In these two approaches, the ATC were calculated assuming that the variation of zonal net export was proportionally distributed on the generators either related to their remaining generation capacities or related to the power they already generate. The zonal approach is common to all the ETSO-EuroPEX methods. Then, the concept of GSK or a similar one is needed for all the methods, even if ETSO and EuroPEX do not explicitly formulate this need. With the GSK matrix, one can then establish (2) between the real PTDF matrix and a zonal PTDF matrix reflecting the definition of market zones.

$P T D F_{\text {zonal }}=G S K * P T D F_{\text {nodal }}$

The GSK matrix may vary between two exports situations. For instance, between two very different export situations, one can expect that more generators will participate in the situation where the export value is the highest. More generators will then be considered in the definition of the GSK matrix in this situation.

A precise evaluation of the GSK matrix is then fundamental. Indeed, if the GSK matrix is wrong, the TSOs cannot ensure that observing contractual congestion in the market clearing corresponds to real physical congestion. An error in the GSK matrix can imply an over- or under-use of the network during contractual congestion. The accuracy of the GSK matrix is also critical for the efficiency of the European congestion management schemes. This point is all the more essential that the regulators may face an asymmetry of information to evaluate the accuracy of the GSK matrix as the TSOs have discretion on setting this matrix.

\section{2) Differences between the ETSO-EuroPEX methods}

Besides the characteristics that make each method different from each other, one particular characteristic allows us to classify the ETSO-EuroPEX methods in two categories, $1^{\circ}$ the ATC-based methods, and $2^{\circ}$ the flow-based method. Obviously, for the two methods namely ATC and Combined ATC, the cross-border capacities are defined relying on the concept of ATC. The other method we study in this paper is the Critical Branch flow-based and as so is classified in the flow-based category. This difference is important because it will force us to model differently the cross-border exchanges for these two categories. For the ATC-based methods, we use a classical equation for any transfer of matter (as water or gas) to link the net (nodal or zonal) production with the interconnection flows. For the flow-based method, we resort on a PTDF (Power Transfer Distribution Factors) matrix that links the net (nodal or zonal) production with the flows on the network lines or interconnections.

The other differences among the ETSO-EuroPEX methods make them different from each other. The two ATC-based methods are different because different assumptions are used to calculate the values of ATC. $1^{\circ}$ The ATC are calculated assuming that the available physical capacity on each line is equally shared among the exchanges between adjacent zones. $2^{\circ}$ The Combined ATC are calculated giving priority to a zone for export. $3^{\circ}$ The Critical Branch flow-based method considers the constraints on each individual line.

\section{MODEL OF THE ETSO-EUROPEX METHOD}

From the similarities and differences of the ETSO-EuroPEX methods, we can now develop a standardised model while also integrating the particularities of each method. We first model the ATC-based methods and then the flow-based ones.

\section{A. A uniform model for the ATC-based methods}

Equations (3) to (5) model an implicit ATC-based congestion management scheme.

$$
\begin{aligned}
& \min \sum_{\text {ithe bids }} C_{i}\left(\Delta P_{i}\right) \\
& \text { s. t. }\left\{\begin{array}{l}
\forall z \text { zone, } \sum_{i \in z} \Delta P_{i}+\sum_{z^{\prime}} T_{z z^{\prime}}=0 \\
\forall\left(z, z^{\prime}\right) \text { a couple of zones, }-A T C_{z^{\prime} z} \leq T_{z z^{\prime}} \leq A T C_{z z^{\prime}}
\end{array}\right.
\end{aligned}
$$

Equation (3) is the objective function minimising social cost resulting from the change in generation pattern after the day- 
ahead market clearing. Equation (4) illustrates the equality constraints for each zone. The change in the net export situation of a zone $z$ is equal to the sum of cross-border exchanges with the neighbouring zones $z$ '. And Equation (5) stands for the ATC limits.

The different ATC-based methods have in common the three following steps of the calculation of ATC. $1^{\circ}$ A nodal base case for generation and load allows to calculate the base case power flow $F_{i j}{ }^{\circ}$ flowing through the line between nodes $i$ and $j$. Only a subset of lines that may be congested is monitored. $2^{\circ}$ The TSO must estimate the GSK matrix. $3^{\circ}$ The coefficient $P T D F_{i j, z z}$, defines the additional flow on line $i j$ for an exchange between the two zones $z$ and $z$ '. Ref. [7] shows that the capacity really available $F_{i j, z z}{ }^{r e}$. $a v$. on line $i j$ for an exchange between zones $z$ and $z$ ' is given by:

$F_{i j, z z^{\prime}}^{\text {re. } a v .}=\left(F_{i j}^{\max }-\operatorname{sign}\left(P T D F_{i j, z z^{\prime}}\right) F_{i j}{ }^{\circ}\right)$

The different ATC-based methods are then characterised by the assumption used to share the available capacity of the monitored lines among the different cross-border exchanges.

1) $\mathrm{ATC}$

The first ATC-based method that we study differs from the other ATC-based methods by the following assumption: the available physical capacity of each powerline $i j$ is equally shared among the $k$ different cross-border exchanges that influence the flow on this line [8]. Ref. [7] presents this method and we recall it with the three following equations.

$F_{i j, z z^{\prime}}{ }^{\text {available }}=\left(F_{i j}{ }^{\max }-\operatorname{sign}\left(P T D F_{i j, z z^{\prime}}\right) F_{i j}{ }^{\circ}\right) / k$

$E x_{z z, i j}{ }^{\max }=\left(F_{i j}^{\max }-\operatorname{sign}\left(P T D F_{i j, z z}\right) F_{i j}{ }^{\circ}\right) /\left(k .\left|P T D F_{i j, z z}\right|\right)$

$A T C_{z z^{\prime}}=\min \left\{\left(F_{i j}^{\max }-\operatorname{sign}\left(P T D F_{i j, z z^{\prime}}\right) F_{i j}{ }^{\circ}\right) /\left(k .\left|P T D F_{i j, z z^{\prime}}\right|\right), i j\right\}$

Where $F_{i j, z z}{ }^{\text {available }}$ is the available physical capacity on line $i j$ for exchanges between the zones $z$ and $z$ ' in this method.

Equation (7) mathematically expressed the above mentioned assumption. Other things equal, (8) gives the maximal exchange $E x_{z z, i j} \max ^{\operatorname{mat}}$ been the zones $z$ and $z$ ' that can constrain line $i j$ (while assuming the other lines cannot be congested). Eventually, (9) gives the value of $A T C_{z z}$, which is the minimal value of $E x_{z z}, i j=\max$ as soon as a line is constrained.

Because of the assumption about sharing the available capacity of line to saturate the interconnections, it is unlikely that a line be really congested when the cross-border exchanges reach the limit set by these ATC.

2) Combined ATC

The Combined ATC stands for a whole family of definition of ATC-based cross-border capacities. We present here an example where the Combined ATC are defined to maximise in priority the exports of a given zone $z$. For this zone, we then compute the optimisation described by (10) to (12).

$A T C_{z z}{ }^{\prime}{ }^{\text {min }}$ is a minimal value that can possibly be imposed on some borders because it can be politically difficult to present zero as a maximal value for some cross-borders exchanges.

The ATC corresponding to the exports from the zones other than the $z$ zone are calculated considering that the export exchanges from the priority zone $z$ must always be able to flow through the network. The export exchanges from zone $z$ are then included in a base case specially used to calculate the ATC from the other zones. It may then be needed to give each zone a priority. We then realise the same process as the one described here.

$\max \sum_{z^{\prime}} A T C_{z z^{\prime}}$
s. t. $\left\{\begin{array}{l}\forall l \text { line, }\left|P T D F_{l, z z^{\prime}} A T C_{z z^{\prime}}\right| \leq F_{l}^{\max } \\ \forall z^{\prime} \text { zone, } A T C_{z z^{\prime}} \geq A T C_{z z^{\prime}}^{\min }\end{array}\right.$

These iterative choices of priority for export from the different zones are similar to define approximately a zonal merit order. The zone with the highest priority to export is supposed to have the smallest price, etc. If the realised market equilibrium is to far from the estimation of the zonal merit order established while computing the Combined ATC, it is possible to have a contractual congestion while the network is far to be physically congested. To overcome this difficulty, it is possible to calculate every set of Combined ATC anticipating every order of priority for export from the different market zones. Each set of Combined ATC would then be tested while calculating the market equilibrium and the retained set of Combined ATC would then be the one which maximises the social surplus.

A major difficulty still remains in this method. It is a problem that the TSO as a monopoly has to anticipate the market outcome through the priority order of export from zones. Even if the TSO is regulated. Besides, it is not sure that the TSO himself wants to do this work: it is out of his core business and it places him in a delicate situation vis-à-vis the network users.

\section{B. A uniform model for flow-based methods}

Equations (13) to (15) model an implicit flow-based congestion management scheme.

$$
\begin{aligned}
& \min \sum_{i \text { the bids }} C_{i}\left(\Delta P_{i}\right) \\
& \text { s. t. }\left\{\begin{array}{l}
\sum_{i} \Delta P_{i}=0 \\
\forall l \text { line or border, }-F_{l}^{\text {min }} \leq P T D F_{l} \cdot[P+\Delta P] \leq F_{l}^{\text {max }}
\end{array}\right.
\end{aligned}
$$

Equation (13) is the objective function of our problem. Equation (14) illustrates the global equality constraint between generation and load. Equation (15) expresses the constraints on flows. The different flow-based methods differ from each other depending on the form of this last equation where flows can be considered aggregated. However, we consider here only the Critical Branch flow-based. 
1) Critical Branch flow-based method

Contrary to the other ETSO-EuroPEX methods, the Critical Branch method distinctively considers the constraints on the individual lines. As a result, in this method, there is no need to define aggregated transmission capacity. However, it is assumed that the influence of generation on flows is zonal, like in the other ETSO-EuroPEX methods. The TSOs then compute a GSK matrix. One can then model this method only changing (15) by (16) in the uniform framework for the flowbased method that we presented just above.

$$
\forall \text { line } l,\left[P T D F_{l}^{\text {nodal }}\right] \cdot[G S K] \cdot\left[P_{\text {base }}+\Delta P\right] \leq F_{l}^{\max }
$$

The constrained lines being explicitly identified gives three main advantages to this method. First, there is no need of assumptions to anticipate the use of infrastructures after the market clearing, neither through a sharing assumption nor through an assumption about the market outcome itself. Second, this method is more transparent than the other ETSOEuroPEX methods because the constraints are not aggregated. Lastly, as this method is very close to nodal pricing, it should lead to a more efficient use of the network, although the effect of the zonal view must be evaluated on economic efficiency and on network security.

\section{EVALUATION AND NUMERICAL COMPARISON}

Now we evaluate the ETSO-EuroPEX methods that we modelled above. First, we verify that the flows resulting from the market outcome respect the real constraints on powerlines. Second, we measure economic efficiency through the social cost on the network presented in II. When needed, we use the GSK matrix presented in III.B.1.

This section is organised as follow. Each subsection studies each of the three main ETSO-EuroPEX methods. We calculate a) the exchange capacities, b) the market equilibrium, and c) the real powerflows resulting from the market outcome.

\section{A. $A T C$}

We evaluate in this subsection the ATC method applying the model of IV.A.1) to the base case in II. To calculate the value of ATC on this system (see table II), the physical available capacity on each line must be shared in 3 because the flow on each line is influenced by three exchanges. For instance, the flow on line 6-7 from 7 to 6 is influenced by the cross-border exchanges from 1 to 2,2 to 3 and 3 to 1 . As a consequence, when the maximal values of ATC are reached, the contractual congestion of a line means that only a third of its available capacity is congested.

TABLE II ATC VALUES
\begin{tabular}{|r|c|c|c|c|c|c|}
\hline ATC & $1-2$ & $1-3$ & $2-3$ & $2-1$ & $3-1$ & $3-2$ \\
\hline MW & 4 & 60 & 1 & 83 & 1 & 39 \\
\hline Line with contractual congestion & $6-7$ & $3-6$ & $6-7$ & $1-3$ & $6-7$ & $3-6$ \\
\hline
\end{tabular}

When the above ATC values are transmitted to the market, the exchanges are constrained by the ATC 1-2, 1-3 and 2-3.
The zonal market prices are then $1^{\circ} 35 € / \mathrm{MWh}, 2^{\circ} 39 € / \mathrm{MWh}$ and $3^{\circ} 58 € / \mathrm{MWh}$. These prices are quite different from the optimal prices that are at maximum $52 € / \mathrm{MWh}$.

The flows resulting from the market outcome don't congest the power lines. This is because of the sharing assumption. The physical congestion of a line can then happen only if the market simultaneously requires that every exchange saturates this line. This situation is very unlikely as the ATC are limited as soon as the first constraint is reached. In our case, it is possible to congest physically only line 6-7.

Since there is a difference in zone prices whereas the network is not congested, the market outcome is suboptimal with a social overcost of $1794 €$ compared to the optimum with nodal pricing.

\section{B. Combined ATC}

We evaluate in this subsection the combined ATC method applying the model of IV.A.2) to the base case in II. We assume that the TSO sets a minimal value of $1 \mathrm{MW}$ to $\mathrm{ATC}_{2-3}$ and $\mathrm{ATC}_{3-2}$. We then obtain the values of ATC in table III assuming that zone 1 has priority for export. The $\mathrm{ATC}_{2-1}$ and $\mathrm{ATC}_{3-1}$ are null because some lines are already congested by the priority exchanges.

TABLE III VALUES OF COMBINED ATC WITH PRIORITY TO ZONE 1

\begin{tabular}{|c|c|c|c|c|c|c|}
\hline ATC & $1-2$ & $1-3$ & $2-3$ & $2-1$ & $3-1$ & $3-2$ \\
\hline MW & 7 & 178 & 1 & 0 & 0 & 1 \\
\hline Line with contractual congestion & $3-6$ & $3-6$ & set & $3-6$ & $6-7$ & set \\
\hline
\end{tabular}

When the above ATC values are transmitted to the market, the exchanges are constrained by the ATC 1-2, 1-3 and 2-3. The zonal market prices are then $1^{\circ} 41 € / \mathrm{MWh}, 2^{\circ} 40 € / \mathrm{MWh}$ and $3^{\circ} 48 € / \mathrm{MWh}$. These prices are quite close to the prices from nodal pricing. Moreover, line 3-6 is the only one to be congested. The market equilibrium with the Combined ATC with priority to zone 1 is very close to the optimum with nodal pricing because the social overcost is only $9 €$.

If now, we assume that the TSO makes a mistake in anticipating the market outcome, he may give priority to export from zone 2. Table IV then gives the new values of ATC. The priority given to the export from zone 2, the possible exports from zone 1 are smaller.

TABLE IV VALUES OF COMBINED ATC WITH PRIORITY TO ZONE 2

\begin{tabular}{|c|c|c|c|c|c|c|}
\hline ATC & $1-2$ & $1-3$ & $2-3$ & $2-1$ & $3-1$ & 3-2 \\
\hline MW & 7 & 48 & 1 & 247 & 0 & 1 \\
\hline Line with contractual congestion & $6-7$ & $3-6$ & set & $1-3$ & $1-3$ & set \\
\hline
\end{tabular}

When the above ATC values are transmitted to the market, the cross-border exchanges are constrained by the ATC 1-2, 1-3 and 2-3. The market price of each zone is then $1^{\circ} 34 € / \mathrm{MWh}, 2^{\circ} 39 € / \mathrm{MWh}$ and $3^{\circ} 59 € / \mathrm{MWh}$. With this market equilibrium, no line is congested. This is because the priority is given to the export from zone 2 while zone 2 is not the cheapest one. We obtain similar results if zone 3 has priority. Since there is difference in zone prices whereas the network is not congested, the market outcome is suboptimal with a social overcost of $2069 €$ compared to the optimum 
with nodal pricing. The performances of the Combined ATC method then depend a lot on the assumption giving priority to a zone for export.

\section{Critical Branch flow-based}

We evaluate in this subsection the Critical Branch flowbased method applying the model of IV.B.1) to the base case in II. With this method, we obtain the following equilibrium prices, $1^{\circ} 42 € / \mathrm{MWh}, 2^{\circ} 39 € / \mathrm{MWh}, 3^{\circ} 48 € / \mathrm{MWh}$ which are quite close to the prices with nodal pricing. The market equilibrium is close to the optimum with nodal pricing because the social overcost is only $17 €$.

Line 3 to 6 constrains the cross-border exchanges. Zone 1 exports $191 \mathrm{MW}$ compared to base case (where there is no cross-border exchange). Zone 2 imports $8 \mathrm{MW}$. And zone 3 imports $183 \mathrm{MW}$. The flow on line 3-6 is higher than the maximal capacity by $0.3 \mathrm{MW}$. The flows can exceed the limit of capacity because of the zonal approximation that consists in estimating the GSK matrix.

The GSK matrix is only an estimation of the participation of generators to the variation of the net export situation of their zone. Once the market equilibrium is established with the Critical Branch flow-based method, it is possible that the realised participation of generators to the net export does not correspond exactly to the anticipated GSK matrix. In this situation, the application of the inaccurate GSK matrix distorts the capacity constraints in the Critical Branch flow-based method. Estimating precisely the GSK matrix corresponding to the reaction of generators to the market price is then important so that the network security can be respected with the Critical Branch flow-based method. Otherwise, the TSO may have to redispatch generation in real time. We haven't seen this problem for the other ETSO-EuroPEX methods because the constraints are not so tight in the other methods.

The Critical Branch flow-based method has also the advantage to reveal precisely where the constrained lines are on the network because the limits on the lines are directly transmitted to the market. The TSOs and the regulators can then directly target their actions toward the lines that are identified as the ones constraining the cross-border exchanges.

Nevertheless, the Critical Branch method has similar drawbacks compared to the other ETSO-EuroPEX methods. First, it is always needed to anticipate to some extent the market equilibrium in order to insert in the Critical Branch method an accurate estimation of the GSK matrix. Second, this method does not always ensure an optimal treatment of constraints on lines that are in a price zone. Indeed, if this internal congestion can be relieved by an internal generator and another one that can be external or internal to the zone, in all the ETSO-EuroPEX methods, this second generator will be systematically external to the zone. Indeed, from the point of view of the algorithm of the Critical Branch method, the joint action of two generators in the same zone (one of them increases its production while the other one decreases it) on any line is null because these two generators have the same zonal PTDF.

\section{CONCLUSIONS AND FUTURE RESEARCHES}

In this paper, we have modelled and evaluated the technical and economic efficiency of the main methods proposed by ETSO and EuroPEX to allocate interconnection capacity in Europe. We can draw two main conclusions from the models and analyses of the ATC- and flow-based allocation methods. Firstly, our results about the relative efficiency of the allocation methods are more classical than the preliminary conclusions of the Implementation Study [4]. The allocation methods are all the more efficient as the Kirchhoff laws are integrated more precisely in the market clearing. In particular, the gains from a flow-based allocation method are mainly generated when the transmission capacities are precisely related to powerlines rather than related to aggregated border capacities only. Secondly, we find that the paradigm according to which the price zones fit the national boundaries and shared by all the allocation methods of ETSO and Europex has important consequences. The zonal vision of the grid may result in the capacity limits of powerlines being exceeded. Besides, this zonal vision of the grid allows to manage internal congestions with only limited efficiency.

Considering our conclusion, future researches will then focus on two points. First, we will evaluate the influence of the accuracy of the GSK matrix on the technical and economic efficiency of the ETSO-EuroPEX methods. Second, we will study the incentives of the generators and TSOs to make the market converge toward this estimation of the market outcome.

\section{REFERENCES}

[1] ETSO and EuroPEX. Development and Implementation of a coordinated model for regional and inter-regional congestion management. Interim report. April 2008. www.rte-france.com

[2] DGComp (10 Jan. 2007), DG Competition report on energy sector inquiry, SEC(2006)1724, http://ec.europa.eu

[3] European Commission (EC) 2006. COMMISSION DECISION of 9 November 2006 amending the Annex to Regulation (EC) No 1228/2003 on conditions for access to the network for cross-border exchanges in electricity $(2006 / 770 / \mathrm{EC})$

[4] APX, Belpex, Cegedel, EEX, Elia, EnBW, Eon Netz, Powernext, RTE, RWE, Tennet. "Implementation Study. A report for the MoU signatories on the design of the market coupling solution in the Central West European (CWE) region". August 2008.

[5] Bompard E., Correia P, Gross G., Amelin M. "A comparative analysis of congestion management schemes under a unified framework", IEEE Trans. on Power Sys., 18, 346-352, Feb. 2003.

[6] ETSO. "Definitions of Transfer Capacities in Liberalised Electricity Markets". April 2001. www.etso-net.org

[7] Rious V., Usaola J., Saguan M., et al. "Assessing Available Transfer Capacity on a Realistic European Network: Impact of Assumptions on Wind Power Generation", in proc. 1st Int. scient. conf. "Building Networks for a Brighter Future", 10-12 Nov. 2008

[8] RTE http://www.rte-france.com/htm/fr/offre/offre_inter_capa.jsp 